TRANSACTIONS OF THE

AMERICAN MATHEMATICAL SOCIETY

Volume 356, Number 4, Pages 1281-1290

S 0002-9947(03)03380-4

Article electronically published on November 25, 2003

\title{
RANDOM GAPS UNDER CH
}

\author{
JAMES HIRSCHORN
}

\begin{abstract}
It is proved that if the Continuum Hypothesis is true, then one random real always produces a destructible $\left(\omega_{1}, \omega_{1}\right)$ gap.
\end{abstract}

\section{INTRODUCTION}

Recall that for a Boolean algebra $(\mathcal{B}, \leq)$, for a pair of types $(\psi, \theta)$ a pair $(A, B)$ of subsets of $\mathcal{B}$ is a $(\psi, \theta)$ pregap in $(\mathcal{B}, \leq)$ if

(a) $A$ and $B$ are linearly ordered by $\leq$,

(b) $(A, \leq)$ has type $\psi$ and $(B, \leq)$ has type $\theta$,

(c) $A \perp B$, i.e. $a \cdot b=0$ for all $a \in A$ and $b \in B$.

The pregap is a gap if moreover

(d) there is no $c \in \mathcal{B}$ such that $a \leq c$ for all $a \in A$, and $b \leq-c$ for all $b \in B$. Such an element $c$ is said to interpolate $A$ and $B$.

One of the more striking early discoveries in set theory is Hausdorff's construction Hau36] of an $\left(\omega_{1}, \omega_{1}\right)$ gap in the Boolean algebra $\left(\mathcal{P}(\mathbb{N}) /\right.$ Fin, $\left.\subseteq^{*}\right)$, where $\mathbb{N}=$ $\{0,1, \ldots\}$ denotes the set of all nonnegative integers. Recall that for $a, b \subseteq \mathbb{N}$,

(e) $[a] \subset^{*}[b]$ iff $a \backslash b$ is finite and $b \backslash a$ is infinite, and $[a] \subseteq^{*}[b]$ iff $[a] \subset^{*}[b]$ or $[a]=[b]$. Lifting this relation to $\mathcal{P}(\mathbb{N})$, we get $a \subseteq^{*} b$ iff $a \backslash b$ is finite. As is usual when working with gaps in $\mathcal{P}(\mathbb{N}) /$ Fin we identify members of $\mathcal{P}(\mathbb{N}) /$ Fin with representatives of their equivalence classes. Thus for a pair of ordinals $(\gamma, \delta)$, we take as a $(\gamma, \delta)$ pregap in $\mathcal{P}(\mathbb{N}) /$ Fin, a pair $(\vec{a}, \vec{b})$ of sequences of subsets of $\mathbb{N}$ such that

(f) $a_{\alpha} \subset^{*} a_{\beta}$ for all $\alpha<\beta<\gamma$,

(g) $b_{\alpha} \subset^{*} b_{\beta}$ for all $\alpha<\beta<\delta$,

(h) $a_{\alpha} \cap b_{\beta}$ is finite for all $\alpha, \beta$,

and $c \subseteq \mathbb{N}$ interpolates $\vec{a}$ and $\vec{b}$ accordingly. In the case where $(A, B)$ is a symmetric pregap, i.e. $\gamma=\delta$, it will be seen to be useful to choose the representatives so that

(i) $a_{\alpha} \cap b_{\alpha}=\emptyset$ for all $\alpha$.

One of the more interesting phenomenon associated with gaps in $\mathcal{P}(\mathbb{N}) /$ Fin is the phenomenon of destructibility. That is, the possibility of interpolating a gap in some forcing extension (and thus rendering it a nongap) while preserving its types. In other words, for a pair $(\kappa, \lambda)$ of cardinals, a $(\kappa, \lambda)$ pregap $(A, B)$ is destructible if there is a poset $\mathcal{P}$ preserving all cardinals less than or equal to $\max \{\kappa, \lambda\}$ which forces a $c \in \mathcal{P}(\mathbb{N}) /$ Fin interpolating $A$ and $B$.

Received by the editors October 1, 2001.

2000 Mathematics Subject Classification. Primary 03E05; Secondary 03E40, 03E50, $28 E 15$.

Key words and phrases. Gap, destructible gap, random real, Continuum Hypothesis. 
It is well known that the existence of a destructible $\left(\omega_{1}, \omega_{1}\right)$ gap is undecidable from the ZFC axioms. Kunen Kun76a, showed that $\mathfrak{m}>\aleph_{1}$ (i.e. $\mathrm{MA}_{\aleph_{1}}$ ) implies that all $\left(\omega_{1}, \omega_{1}\right)$ gaps are indestructible (see also [Sch93]). On the other hand the first destructible $\left(\omega_{1}, \omega_{1}\right)$ gap was constructed by Laver [Lav79] by forcing with finite approximations to an $\left(\omega_{1}, \omega_{1}\right)$ pregap (see also [Sch93]). It was later shown by Todorčević that a destructible $\left(\omega_{1}, \omega_{1}\right)$ gap can be constructed from a diamond sequence (see Dow95). This begged the question of whether the Continuum Hypothesis alone is sufficient for the existence of a destructible gap. This was answered negatively in AT97, where it is also shown that the combinatorial principle $(*)$ is consistent with $\mathrm{CH}$.

Theorem 1 (Abraham-Todorčević $(*))$. All $\left(\omega_{1}, \omega_{1}\right)$ gaps are indestructible.

Furthermore, a destructible $\left(\omega_{1}, \omega_{1}\right)$ gap can be constructed from a Cohen real. Indeed the simplest and most elegant construction of a destructible $\left(\omega_{1}, \omega_{1}\right)$ gap is the following one due to Todorčević. We write $\mathcal{C}$ for the Cohen poset on $2^{<\mathbb{N}}$, and write $\dot{c}$ for the canonical $\mathcal{C}$-name for the Cohen real in $\mathcal{P}(\mathbb{N}) \cong 2^{\mathbb{N}}$. Operations on $\mathcal{P}(\mathbb{N})$ are extended memberwise to subfamilies of $\mathcal{P}(\mathbb{N})$.

Theorem 2 (Todorčević). Suppose that $(\vec{a}, \vec{b})$ is an $\left(\omega_{1}, \omega_{1}\right)$ gap in $\mathcal{P}(\mathbb{N}) /$ Fin. Then

$$
\mathcal{C} \Vdash(\vec{a} \cap \dot{c}, \vec{b} \cap \dot{c}) \text { is a destructible }\left(\omega_{1}, \omega_{1}\right) \text { gap, }
$$

i.e. $(\vec{a} \cap \dot{c}, \vec{b} \cap \dot{c})=\left(\left(a_{\alpha} \cap \dot{c}: \alpha<\omega_{1}\right),\left(b_{\alpha} \cap \dot{c}: \alpha<\omega_{1}\right)\right)$.

Proof. See [TF95, Theorem 9.3].

This construction can be viewed as the simplest gap introduced by a Cohen real. A question which then naturally arises is what happens if $c$ is replaced with a random real? Indeed it is asked in [TF95, Chapter 9] whether the analogue of Theorem 2 is true for a random real, and this was answered negatively in Hir01. with the following result. We let $\mathcal{R}$ denote the atomless separable measure algebra (i.e. the poset for adding one random real), and let $\dot{r}$ be the canonical $\mathcal{R}$-name for the random real in the Haar measure space $2^{\mathbb{N}}$ identified with $\mathcal{P}(\mathbb{N})$. Note that the combinatorial principle $\left(\star_{c}\right)$ is also consistent with $\mathrm{CH}$, as established in Hir00a.

Theorem $3\left(\mathfrak{m}>\aleph_{1}\right.$ or $(*)$ or $\left.\left(\star_{c}\right)\right)$. For every $\left(\omega_{1}, \omega_{1}\right)$ gap $(\vec{a}, \vec{b})$, $\mathcal{R}$ forces that $(\vec{a} \cap \dot{r}, \vec{b} \cap \dot{r})$ is indestructible.

More generally, adding a random real need not introduce any destructible gaps at all (see [Hir01):

Theorem $4\left(\mathfrak{m}>\aleph_{1}\right)$. $\mathcal{R}$ forces that all $\left(\omega_{1}, \omega_{1}\right)$ gaps are indestructible.

It is an open problem ${ }^{1}$ whether this generalizes to arbitrary measure algebras (i.e. the extension by arbitrarily many random reals).

Now while neither $\mathrm{CH}$ nor a random real alone suffices to obtain a destructible gap, we prove here that both together do yield a destructible gap. Indeed the purpose of this note is to prove the following theorem.

Theorem $5(\mathrm{CH}) \cdot \mathcal{R} \Vdash$ there exists a destructible $\left(\omega_{1}, \omega_{1}\right)$ gap.

\footnotetext{
${ }^{1}$ Added in proof: The author has recently obtained a negative answer in [Hir03].
} 
However, we point out that by Theorem[3 this cannot be obtained by considering the simplest random gap (i.e. the random real analogue of the construction in Theorem (2). The destructible gap is obtained by considering the "next simplest" random gap. That is, we prove that assuming $\mathrm{CH}$, there exists a gap $(\vec{a}, \vec{b})$ and an $\mathcal{R}$-name $\dot{s}$ for a subset of $\mathbb{N}$ such that

$$
\mathcal{R} \Vdash(\vec{a} \cap \dot{s}, \vec{b} \cap \dot{s}) \text { is a destructible gap. }
$$

We do not however directly construct these objects.

Definition 6. For a function $f$ whose range consists entirely of finite sets, define

$$
\mathbb{R}(f)=\prod_{z \in \operatorname{dom}(f)} f(z)
$$

For example, for the identity function $i: \mathbb{N}^{+} \rightarrow \mathbb{N}^{+}\left(\mathbb{N}^{+}=\{1,2, \ldots\}\right.$ denotes the positive integers), $\mathbb{R}(i)$ consists of all sequences $\left(k_{n}: n \in \mathbb{N}^{+}\right)$such that $k_{n}<n$ for all $n$. We give $\mathbb{R}(f)$ the product topology, and it is equipped with the product measure $\nu=\prod_{z \in \operatorname{dom}(f)} \nu_{z}$ where $\nu_{z}(\{w\})=1 /|f(z)|$ for all $w \in f(z)$.

Notation. Let $h: \mathbb{N} \times \mathbb{N} \rightarrow \mathbb{N}$ be fixed. For $a \subseteq \mathbb{N} \times \mathbb{N}$, we let

$$
[a]=\bigcup_{(m, n) \in a}\{(m, n)\} \times h(m, n) .
$$

Note that every element of $\mathbb{R}(h)$ is a subset of $[\mathbb{N} \times \mathbb{N}]$.

Instead of the objects in (1), we fix a function $h: \mathbb{N}^{2} \rightarrow \mathbb{N}$ and construct a gap $(\vec{a}, \vec{b})$ in $\mathcal{P}([\mathbb{N} \times \mathbb{N}]) /$ Fin, i.e. $a_{\alpha}, b_{\alpha} \subseteq[\mathbb{N} \times \mathbb{N}]$ for all $\alpha$, such that

$$
\mathcal{R} \Vdash\left(\vec{a} \cap \dot{r}_{\mathbb{R}(h)}, \vec{b} \cap \dot{r}_{\mathbb{R}(h)}\right) \text { is a destructible gap, }
$$

where $\dot{r}_{\mathbb{R}(h)}$ denotes an $\mathcal{R}$-name for the random real in the measure space $\mathbb{R}(h)$. To see that the objects in (2) yield those in (1), fix a bijection $g: \mathbb{N} \rightarrow[\mathbb{N} \times \mathbb{N}]$. Then $g$ induces a homeomorphism $\Phi_{g}: \mathcal{P}([\mathbb{N} \times \mathbb{N}]) \rightarrow \mathcal{P}(\mathbb{N})$ via

$$
\Phi_{g}(d)=g^{-1}(d) \text {. }
$$

Furthermore it is clear that $\Phi_{g}$ induces an isomorphism $\widetilde{\Phi}_{g}: \mathcal{P}([\mathbb{N} \times \mathbb{N}]) /$ Fin $\rightarrow$ $\mathcal{P}(\mathbb{N}) /$ Fin via $\widetilde{\Phi}_{g}([d])=\left[\Phi_{g}(d)\right]$ (here square brackets denote the equivalence class). Therefore $\left(\Phi_{g}^{\prime \prime}(\vec{a}), \Phi_{g}^{\prime \prime}(\vec{b})\right)$ is a pregap in $\mathcal{P}(\mathbb{N}) /$ Fin and $\Phi_{g}\left(\dot{r}_{\mathbb{R}(h)}\right)$ is an $\mathcal{R}$-name for a subset of $\mathbb{N}$ such that

$$
\left(\Phi_{g}^{\prime \prime}(\vec{a}) \cap \Phi_{g}\left(\dot{r}_{\mathbb{R}(h)}\right), \Phi_{g}^{\prime \prime}(\vec{b}) \cap \Phi_{g}\left(\dot{r}_{\mathbb{R}(h)}\right)\right)
$$

is a destructible gap with probability one.

It is instructive to note that this is not the first instance of such a construction involving both $\mathrm{CH}$ and a random real in a measure space of the form $\mathbb{R}(h)$. Indeed, in [Hir00b Example 5.5] there is a $\mathrm{CH}$ construction of a maximal tower in $\mathcal{P}(\mathbb{N}) /$ Fin (i.e. a well-ordered subfamily) such that for some $e: \mathbb{N} \rightarrow \mathbb{N}$ with fast enough growth, when the random real in $\mathbb{R}(e)$ is transferred to $\mathcal{P}(\mathbb{N})$ via the map analogous to $\Phi_{g}$ it extends this tower (i.e. kills its maximality).

There is another motivation for proving Theorem [5 Many analogies exist between $\left(\omega_{1}, \omega_{1}\right)$ gaps and Aronszajn trees (see e.g. AT97). For example, Souslin trees are analogous to destructible gaps because any Souslin tree can be destroyed by an $\omega_{1}$-preserving forcing notion which forces a cofinal branch through the tree. Furthermore, Souslin trees can be constructed from a diamond sequence, and also 
from a Cohen real. Analogously to Kunen's theorem above, $\mathfrak{m}>\aleph_{1}$ implies that all Aronszajn trees are special. Thus destructible $\left(\omega_{1}, \omega_{1}\right)$ gaps correspond to Souslin trees, gaps correspond to Aronszajn trees and indestructible gaps correspond to special Aronszajn trees. However, in [Hir00a] it is proved that $\left(\star_{c}\right)$ implies that all Aronszajn trees are special in any forcing extension by a measure algebra. Thus by adding one random real to a model satisfying $\mathrm{CH}$ and $\left(\star_{c}\right)$, Theorem 5 breaks this analogy between gaps and Aronszajn trees. Indeed, to the author's knowledge this is the first model where all Aronszajn trees are special yet there exists a destructible $\left(\omega_{1}, \omega_{1}\right)$ gap. Theorem 5 also answers negatively a question along these lines of Todorčević in [Tod00, p. 253].

\section{THE MAIN LEMMA}

The following Ramsey theoretic characterization of destructibility is well known. We refer the reader to one of the sources [Woo84, Tod89, Sch93, [TF95, Lemma $9.2]$ for its proof. The results of this section all require that an $\left(\omega_{1}, \omega_{1}\right)$ pregap satisfies condition (i). Note that in the following Lemmas 7 and 8 , a pregap refers to a pregap in $\left(\mathcal{P}(S) /\right.$ Fin, $\left.\subseteq^{*}\right)$ for some countable set $S$.

Lemma 7. For any $\left(\omega_{1}, \omega_{1}\right)$ pregap $(\vec{a}, \vec{b})$ the following are equivalent:

(a) $(\vec{a}, \vec{b})$ is destructible.

(b) For every uncountable $X \subseteq \omega_{1}$ there exist $\alpha \neq \beta$ in $X$ such that $\left(a_{\alpha} \cap b_{\beta}\right) \cup$ $\left(a_{\beta} \cap b_{\alpha}\right)=\emptyset$.

(c) There is a poset with the ccc which interpolates $(\vec{a}, \vec{b})$.

The following sufficient condition for an $\mathcal{R}$-name for an $\left(\omega_{1}, \omega_{1}\right)$ pregap to name a destructible gap with positive probability is from [Hir01. Note that it has no analogue for the trivial measure algebra, i.e. if there is an uncountable $X \subseteq \omega_{1}$ such that $a_{\alpha} \cap b_{\beta}=\emptyset$ for all $\alpha, \beta \in X$, then it is clear that $(\vec{a}, \vec{b})$ is not a gap. On the other hand, we are going to prove Theorem 5 by constructing an $\mathcal{R}$-name for a gap satisfying this condition.

We use standard notation for Boolean algebras, and thus for a sentence $\phi$ in the forcing language of a given Boolean algebra, $\|\phi\|$ denotes the Boolean probability that $\phi$ is true. The minimum and maximum elements of a Boolean algebra are denoted by 0 and 1 , respectively. Note that we actually force with the poset $\mathcal{R}^{+}=$ $\mathcal{R} \backslash\{0\}$ of positive elements of $\mathcal{R}$. As usual, for each $x \in \mathcal{R}, \mathcal{R}_{x}$ denotes the Boolean subalgebra $\{y \in \mathcal{R}: y \leq x\}$.

Lemma 8. Let $(\vec{a}, \vec{b})$ be an $\mathcal{R}$-name for an $\left(\omega_{1}, \omega_{1}\right)$ pregap. Then for every $x \in$ $\mathcal{R}^{+}$, if there exists an uncountable $X \subseteq \omega_{1}$ and a $\rho<1$ such that

$$
\mu\left(x \cdot\left(\left\|\dot{a}_{\alpha} \cap \dot{b}_{\beta} \neq \emptyset\right\|+\left\|\dot{a}_{\beta} \cap \dot{b}_{\alpha} \neq \emptyset\right\|\right)\right) \leq \rho \cdot \mu(x) \quad \text { for all } \alpha, \beta \in X,
$$

then $x \cdot \|(\vec{a}, \vec{b})$ is destructible $\| \neq 0$.

Proof. Given $x \in \mathcal{R}^{+}$, assume that $x$ forces that $(\vec{a}, \vec{b})$ is indestructible. Suppose we are given an uncountable $X \subseteq \omega_{1}$ and $\rho<1$. By Lemma 7 there is an $\mathcal{R}$-name $\dot{Y}$ for an uncountable subset of $X$ such that

$$
x \leq\left\|\forall \alpha, \beta \in \dot{Y} \quad \alpha \neq \beta \rightarrow\left(\dot{a}_{\alpha} \cap \dot{b}_{\beta}\right) \cup\left(\dot{a}_{\beta} \cap \dot{b}_{\alpha}\right) \neq \emptyset\right\| .
$$


Recursively choose for each $\eta<\omega_{1}$ a finite antichain $\left\{y_{\eta}^{0}, \ldots, y_{\eta}^{m_{\eta}-1}\right\} \subseteq \mathcal{R}_{x}^{+}$and $\left\{\alpha(\eta, 0)<\cdots<\alpha\left(\eta, m_{\eta}-1\right)\right\} \subseteq \omega_{1}$ such that

$$
\begin{gathered}
\mu\left(\sum_{i<m_{\eta}} y_{\eta}^{i}\right)>\sqrt{\rho} \cdot \mu(x), \\
y_{\eta}^{i} \leq\|\alpha(\eta, i) \in \dot{Y}\| \quad \text { for all } i<m_{\eta}, \\
\alpha(\eta, 0)>\alpha\left(\xi, m_{\xi}-1\right) \quad \text { for all } \xi<\eta .
\end{gathered}
$$

Find a finite $s_{\eta} \subseteq S$ large enough so that

$$
\begin{array}{ll}
\mu\left(\left\|\dot{a}_{\alpha(\eta, i)} \backslash s_{\eta} \subseteq \dot{a}_{\alpha\left(\eta, m_{\eta}-1\right)}\right\|\right)>1-\frac{(\sqrt{\rho}-\rho) \cdot \mu(x)}{4 m} & \text { for all } i<m_{\eta}, \\
\mu\left(\left\|\dot{b}_{\alpha(\eta, i)} \backslash s_{\eta} \subseteq \dot{b}_{\alpha\left(\eta, m_{\eta}-1\right)}\right\|\right)>1-\frac{(\sqrt{\rho}-\rho) \cdot \mu(x)}{4 m} & \text { for all } i<m_{\eta} .
\end{array}
$$

By going to an uncountable subsequence we may assume that $m_{\eta}=m$ and $s_{\eta}=s$ for all $\eta$. Since $\mathcal{R}$ is separable, which means that the metric space $(\mathcal{R}, \nu)$ where $\nu(a, b)=\mu(a \triangle b)$ is separable, we can find an uncountable $Z \subseteq \omega_{1}$ such that

$$
\mu\left(y_{\xi}^{i} \triangle y_{\eta}^{i}\right) \leq \frac{(\sqrt{\rho}-\rho) \cdot \mu(x)}{4 m} \text { for all } i<m, \text { for all } \xi, \eta \in Z .
$$

Using separability in a similar manner we can further assume that

$$
\mu\left(\left\|\dot{b}_{\alpha(\xi, i)} \cap s=\dot{b}_{\alpha(\eta, i)} \cap s\right\|\right)>1-\frac{(\sqrt{\rho}-\rho) \cdot \mu(x)}{4 m} \text { for all } i<m .
$$

Take any $\xi \neq \eta$ in $Z$. We claim that

(12) $\mu\left(x \cdot\left(\left\|\dot{a}_{\alpha(\xi, m-1)} \cap \dot{b}_{\alpha(\eta, m-1)} \neq \emptyset\right\|+\left\|\dot{a}_{\alpha(\eta, m-1)} \cap \dot{b}_{\alpha(\xi, m-1)} \neq \emptyset\right\|\right)\right)>\rho \cdot \mu(x)$.

By (4), (6) and (7),

$$
y_{\xi}^{i} \cdot y_{\eta}^{i} \leq\left\|\dot{a}_{\alpha(\xi, i)} \cap \dot{b}_{\alpha(\eta, i)} \neq \emptyset\right\|+\left\|\dot{a}_{\alpha(\eta, i)} \cap \dot{b}_{\alpha(\xi, i)} \neq \emptyset\right\| \quad \text { for all } i<m .
$$

Since by (i) and (11), $\mu\left(\left\|\left(\dot{a}_{\alpha(\xi, i)} \cap \dot{b}_{\alpha(\eta, i)} \cap s\right) \cup\left(\dot{a}_{\alpha(\eta, i)} \cap \dot{b}_{\alpha(\xi, i)} \cap s\right)=\emptyset\right\|\right)>$ $1-(\sqrt{\rho}-\rho) \cdot \mu(x) / 4 m$ for all $i<m$, it follows from (8) and (9) that

$$
\begin{aligned}
& \mu\left(\sum_{i<m} y_{\xi}^{i} \cdot y_{\eta}^{i}\right)-\frac{3(\sqrt{\rho}-\rho) \cdot \mu(x)}{4} \\
& \quad<\mu\left(\left\|\dot{a}_{\alpha(\xi, m-1)} \cap \dot{b}_{\alpha(\eta, m-1)} \neq \emptyset\right\|+\left\|\dot{a}_{\alpha(\eta, m-1)} \cap \dot{b}_{\alpha(\xi, m-1)} \neq \emptyset\right\|\right) .
\end{aligned}
$$

However, by (5) and (10), $\mu\left(\sum_{i<m} y_{\xi}^{i} \cdot y_{\eta}^{i}\right) \geq \sqrt{\rho} \cdot \mu(x)-(\sqrt{\rho}-\rho) \cdot \mu(x) / 4$, which completes the proof of (12) and hence also the proof of the lemma.

Lemma 8 points to a new phenomenon. Every $\left(\omega_{1}, \omega_{1}\right)$ gap can be frozen, which means that there is a poset which forces that the gap is indestructible (see e.g. the references at the beginning of this section). However, setting $x=1$, the condition in Lemma 8 is clearly upwards absolute for transitive models. Therefore, an $\mathcal{R}$-name for a gap satisfying the hypothesis remains an $\mathcal{R}$-name for a destructible pregap (and possibly a nongap) in any forcing extension, and thus freezing is impossible in this context. Furthermore, using this lemma it is shown in Hir01 that such an $\mathcal{R}$-name $(\vec{a}, \vec{b})$ for a gap can be destroyed by a poset satisfying property $K$, i.e. there is a poset with property $K$ which forces that $\|(\vec{a}, \vec{b})$ is a gap $\| \neq 1$. On the other hand, it is easy to see that a poset with property $K$ can never destroy an $\left(\omega_{1}, \omega_{1}\right)$ gap. 


\section{The CONSTRUCTION}

Preliminarily to the construction we review two notions to be used here. Suppose $\mathcal{U}$ is an ultrafilter on $\mathbb{N}$ and $[f]$ is a member of the ultraproduct $\mathcal{R}^{\mathbb{N}} / \mathcal{U}$ with a representative $f \in \mathcal{R}^{\mathbb{N}}$. Then in $V[\mathcal{G}]$, a forcing extension by $\mathcal{R}$, we consider the limit

$$
\lim _{x \rightarrow \mathcal{G}} \lim _{n \rightarrow \mathcal{U}} \frac{\mu(x \cdot f(n))}{\mu(x)} .
$$

For a function $g: \mathbb{N} \rightarrow \mathbb{R}$, one defines $\lim _{n \rightarrow \mathcal{U}} g(n)=w$ if for every open $G$ about $w$, $\{n: g(n) \in G\} \in \mathcal{U}$. In $V[\mathcal{G}]$, for a function $h: \mathcal{R} \rightarrow \mathbb{R}$, one defines $\lim _{x \rightarrow \mathcal{G}} h(x)=v$ if for every open $H$ about $v$, there is a $y \in \mathcal{G}$ such that $h^{\prime \prime}\{x \in \mathcal{G}: x \leq y\} \subseteq H$. A limit as in 15 first appears in Kunen's paper Kun76b, and has its origins in Solovay's paper [Sol71] (see also [Jec97], [Kan94, §17]). A basic fact which we use is that

$$
y \Vdash \lim _{x \rightarrow \dot{\mathcal{G}}} \lim _{n \rightarrow \breve{\mathcal{U}}} \frac{\mu(x \cdot f(n))}{\mu(x)}>\check{\varepsilon} \quad \text { iff } \quad \lim _{n \rightarrow \mathcal{U}} \frac{\mu(z \cdot f(n))}{\mu(z)}>\varepsilon \quad \text { for all } z \leq y,
$$

and the corresponding statement with $<\varepsilon$ in place of $>\varepsilon$. We refer the reader to one of these expositions for the proof that the limit in (15) is well defined and always exists, and the proof of fact (16).

The other basic fact is that given a sequence $\left(x_{n}: n \in \mathbb{N}\right)$ of elements in $\mathcal{R}$ such that $\mu\left(x_{n}\right) \geq \delta$ for all $n$, for some $\delta>0$,

$$
\mu\left(\|\left\{n: x_{n} \in \dot{\mathcal{G}}\right\} \text { is infinite } \|\right) \geq \delta .
$$

In probabilistic terms this is just another way of saying that for a sequence of events with probability bounded away from zero, there is a positive probability that infinitely many of these events occur. To see that (17) holds, note that $\|\left\{x_{n}: n \in \dot{\mathcal{G}}\right\}$ is infinite $\left\|=\prod_{n=0}^{\infty} \sum_{m=n}^{\infty}\right\| x_{m} \in \dot{\mathcal{G}} \|$.

Fix some function $h: \mathbb{N} \rightarrow \mathbb{N}^{+}$such that

$$
\rho=2 \cdot \sum_{m=0}^{\infty} \frac{1}{h(m)}<1 .
$$

Let $\hat{h}: \mathbb{N} \times \mathbb{N} \rightarrow \mathbb{N}$ be the function where $\hat{h}(m, n)=h(m)$ for all $m, n$. The square bracket notation from the Introduction will refer to this $\hat{h}$. We write $\dot{r}$ for $\dot{r}_{\mathbb{R}(\hat{h})}$.

Before proceeding, we try to give some motivation for the conditions imposed in our construction below. To ensure that we construct an $\mathcal{R}$-name for a destructible gap, we require that for all $\alpha, \beta,\left\|a_{\alpha} \cap b_{\beta} \cap \dot{r} \neq \emptyset\right\|+\left\|a_{\beta} \cap b_{\alpha} \cap \dot{r} \neq \emptyset\right\|$ has measure at most $\rho$. Then by Lemma $8\left(a_{\alpha} \cap \dot{r}, b_{\alpha} \cap \dot{r}: \alpha<\omega_{1}\right)$ will be destructible with positive probability. Thus we also have to ensure that the $\mathcal{R}$-name $(\vec{a} \cap \dot{r}, \vec{b} \cap \dot{r})$ for a pregap is in fact a gap with probability one, which gives the following opposing restriction.

Lemma 9. Let $(\vec{a}, \vec{b})$ be an $\mathcal{R}$-name for a pregap. If $\|(\vec{a}, \vec{b})$ is a gap $\|=1$, then for every uncountable $X \subseteq \omega_{1}$, there exists $\beta \in X$ and an $\mathcal{R}$-name $\dot{\alpha}$ for an ordinal in $X$ such that $\left\|\dot{a}_{\dot{\alpha}} \cap \dot{b}_{\breve{\beta}} \neq \emptyset\right\|=1$.

Proof. Since $\left(\dot{a}_{\alpha}, \dot{b}_{\alpha}: \alpha \in X\right)$ is a gap with probability one, the set named by

$$
\dot{c}=\bigcup_{\alpha \in X} \dot{a}_{\alpha}
$$


interpolates the gap with probability zero, and therefore there is an $\mathcal{R}$-name $\dot{\beta}$ for an ordinal in $X$ such that $\| \dot{b}_{\dot{\beta}} \cap \dot{c}$ is infinite $\|=1$. Thus if $\gamma \in X$ is above the supremum of the set $\{\beta \in X:\|\dot{\beta}=\check{\beta}\| \neq 0\}$, then $\| \dot{b}_{\check{\gamma}} \cap \dot{c}$ is infinite $\|=1$. In particular, $\dot{b}_{\gamma} \cap \dot{c} \neq \emptyset$ with probability one, and therefore there is an $\mathcal{R}$-name $\dot{\alpha}$ for an element of $X$ such that $\dot{a}_{\dot{\alpha}}$ intersects $\dot{b}_{\gamma}$ with probability one.

In particular, by approximating the value of $\dot{\alpha}$ by a finite antichain, this means that finite-to-one intersections must occur with high probability. In other words on any uncountable $X \subseteq \omega_{1}$ there will be a finite $\Gamma \subseteq X$ and a $\beta \in X$ such that $\left\|\bigcup_{\alpha \in \Gamma} a_{\alpha} \cap b_{\beta} \cap \dot{r} \neq \emptyset\right\|$ has measure arbitrarily close to one. Conditions (iii) and (v) together imply that for all $\alpha, \beta$, for every $m \in \mathbb{N}, a_{\alpha} \cap b_{\beta} \cap[\{m\} \times \mathbb{N}]$ has at most one element, and therefore $\left\|a_{\alpha} \cap b_{\beta} \cap[\{m\} \times \mathbb{N}] \cap \dot{r} \neq \emptyset\right\|$ has measure at most $h(m)^{-1}$, guaranteeing that $\left\|a_{\alpha} \cap b_{\beta} \cap \dot{r} \neq \emptyset\right\|+\left\|a_{\beta} \cap b_{\alpha} \cap \dot{r} \neq \emptyset\right\|$ has measure at most $\rho$ (see (21) ). On the other hand, there is nothing preventing the existence of $\alpha_{0}, \ldots, \alpha_{h(m)-1}$ and $n \in \mathbb{N}$ such that $(m, n, i) \in a_{\alpha_{i}}$ for all $i<h(m)$, and a $\beta$ such that $\{(m, n)\} \times h(m) \subseteq b_{\beta}$, in which case $\left\|\bigcup_{i<h(m)} a_{\alpha_{i}} \cap b_{\beta} \cap \dot{r} \neq \emptyset\right\|=1$, because

$$
\| \mid\{(m, n)\}] \cap \dot{r} \mid=1 \|=1
$$

for all $m, n$.

We use $\mathrm{CH}$ to get an enumeration $\left\{\left(\dot{s}_{\alpha}, x_{\alpha}\right): \alpha<\omega_{1}\right\}$ of all pairs $(\dot{s}, x)$ where $\dot{s}$ is an $\mathcal{R}$-name for a subset of $[\mathbb{N} \times \mathbb{N}]$ and $x \in \mathcal{R}^{+}$.

We define $a_{\beta}, b_{\beta} \subseteq[\mathbb{N} \times \mathbb{N}]$ by recursion on $\beta<\omega_{1}$ so that:

(i) $a_{\beta} \cap b_{\beta} \neq \emptyset$,

(ii) for all $\alpha<\beta, a_{\alpha} \subseteq^{*} a_{\beta}$ and $b_{\alpha} \subseteq^{*} b_{\beta}$,

(iii) for all $m, n \in \mathbb{N}, a_{\beta} \cap[\{(m, n)\}]$ has at most one element (i.e. $a_{\beta}$ is a function on some subset of $\mathbb{N} \times \mathbb{N}$ ),

(iv) for all $m \in \mathbb{N}, a_{\beta} \cap[\{m\} \times \mathbb{N}]$ is finite (i.e. $\operatorname{dom}\left(a_{\beta}\right) \in \emptyset \times$ Fin),

(v) for all $m \in \mathbb{N}$, there is at most one $n \in \mathbb{N}$ such that $b_{\beta} \cap[\{(m, n)\}] \neq \emptyset$ (i.e. $\left.\operatorname{dom}\left(b_{\beta}\right) \in \emptyset \times[\mathbb{N}]^{\leq 1}\right)$,

(vi) for infinitely many $m \in \mathbb{N}, b_{\beta} \cap[\{m\} \times \mathbb{N}]=\emptyset$,

(vii) either
(a) $x_{\beta}-\left\|a_{\beta+1} \cap \dot{r} \subseteq^{*} \dot{s}_{\beta}\right\| \neq 0$, or
(b) $x_{\beta}-\left\|b_{\beta+1} \cap \dot{r} \subseteq^{*} \dot{s}_{\beta}^{\mathrm{c}}\right\| \neq 0$.

Assuming for the moment that this is possible, let us see why this yields the desired object. By (i) and (ii), $\left(a_{\alpha}, b_{\alpha}: \alpha<\omega_{1}\right)$ forms a pregap. And for all $\alpha, \beta<\omega_{1}$,

$$
\begin{aligned}
\mu\left(\left\|a_{\alpha} \cap b_{\beta} \cap \dot{r} \neq \emptyset\right\|\right) & \leq \sum_{m=0}^{\infty} \mu\left(\left\|a_{\alpha} \cap b_{\beta} \cap[\{m\} \times \mathbb{N}] \cap \dot{r} \neq \emptyset\right\|\right) \\
& =\sum_{m=0}^{\infty} \max _{n \in \mathbb{N}} \mu\left(\left\|a_{\alpha} \cap b_{\beta} \cap[\{(m, n)\}] \cap \dot{r} \neq \emptyset\right\|\right) \\
& \leq \sum_{m=0}^{\infty} \frac{1}{h(m)}=\frac{\rho}{2}
\end{aligned}
$$

where (v) is used for the equality, and (iii) is used to obtain the following inequality. Therefore $\mu\left(\left\|a_{\alpha} \cap b_{\beta} \cap \dot{r} \neq \emptyset\right\|+\left\|a_{\beta} \cap b_{\alpha} \cap \dot{r} \neq \emptyset\right\|\right) \leq \rho$; hence, Lemma 8 with $x=1$ and $X=\omega_{1}$ entails that $(\vec{a} \cap \dot{r}, \vec{b} \cap \dot{r})$ is destructible with positive probability. It remains to verify that $(\vec{a} \cap \dot{r}, \vec{b} \cap \dot{r})$ is a gap with probability one. However, if there 
were some $x \in \mathcal{R}^{+}$and some $\mathcal{R}$-name $\dot{s}$ for a subset of $[\mathbb{N} \times \mathbb{N}]$ for which

$$
x \Vdash a_{\alpha} \cap \dot{r} \subseteq^{*} \dot{s} \quad \text { and } \quad b_{\alpha} \cap \dot{r} \subseteq^{*} \dot{s}^{\mathrm{c}} \quad \text { for all } \alpha<\omega_{1},
$$

then choosing $\beta$ such that $\left(x_{\beta}, \dot{s}_{\beta}\right)=(x, \dot{s})$ would result in a contradiction with (vii). Note that we can conclude that with probability one, both $\left(a_{\alpha} \cap \dot{r}: \alpha<\omega_{1}\right)$ and $\left(b_{\alpha} \cap \dot{r}: \alpha<\omega_{1}\right)$ have order type $\omega_{1}$ in $\mathcal{P}(\mathbb{N}) /$ Fin. For otherwise some $x \in \mathcal{R}^{+}$ would force that either $a_{\alpha} \cap \dot{r}$ or $b_{\alpha} \cap \dot{r}$ is eventually constant modulo Fin, and then $x$ would force that $(\vec{a} \cap \dot{r}, \vec{b} \cap \dot{r})$ is not a gap.

Now it remains to show how to perform such a construction. Fix a nonprincipal ultrafilter $\mathcal{U}$ on $\mathbb{N}$. We start the recursion by setting $a_{0}=b_{0}=\emptyset$. For a given $\beta<\omega_{1}$ assume that $a_{\alpha}$ and $b_{\alpha}$ have been defined for all $\alpha<\beta$. Suppose that $\beta$ is a successor. Define $g \in \mathcal{R}^{\mathbb{N} \times \mathbb{N}}$ by

$$
g(m, n)=\left\|[\{(m, n)\}] \cap\left(\dot{r} \backslash \dot{s}_{\beta-1}\right) \neq \emptyset\right\| .
$$

First we consider the following case:

Case 1. There exist infinitely many $m \in \mathbb{N}$ such that

$$
x_{\beta-1} \cdot\left\|\lim _{z \rightarrow \dot{\mathcal{G}}} \lim _{n \rightarrow \breve{\mathcal{U}}} \frac{\mu(z \cdot g(m, n))}{\mu(z)}>0\right\| \text { has measure greater than } \frac{\mu\left(x_{\beta-1}\right)}{2} .
$$

Let $\left\{m_{j}\right\}_{j=0}^{\infty}$ be an enumeration without repetition of all $m \in \mathbb{N}$ satisfying (24). We define a sequence $\{u(j)\}_{j=0}^{\infty}$ such that

$$
\begin{gathered}
u(j) \text { is a finite partial function from }\left\{m_{j}\right\} \times \mathbb{N} \text { into } h\left(m_{j}\right), \\
\operatorname{dom}(u(j)) \cap\left(\operatorname{dom}\left(a_{\beta-1}\right) \cup \operatorname{dom}\left(b_{\beta-1}\right)\right)=\emptyset, \\
\mu\left(x_{\beta-1} \cdot\left\|u(j) \cap\left(\dot{r} \backslash \dot{s}_{\beta-1}\right) \neq \emptyset\right\|\right)>\mu\left(x_{\beta-1}\right) / 2 .
\end{gathered}
$$

To see that this is possible, take $j \in \mathbb{N}$. By (24) there is a $y \leq x_{\beta-1}$ and $\varepsilon>0$ such that

$$
\begin{gathered}
\mu(y)>\frac{\mu\left(x_{\beta-1}\right)}{2}, \\
y \Vdash \lim _{z \rightarrow \dot{\mathcal{G}}} \lim _{n \rightarrow \breve{\mathcal{U}}} \frac{\mu\left(z \cdot g\left(m_{j}, n\right)\right)}{\mu(z)}>\varepsilon .
\end{gathered}
$$

We choose $n_{0}<n_{1}<\cdots<n_{i}<\cdots$ so that

$$
\left(m_{j}, n_{i}\right) \notin \operatorname{dom}\left(a_{\beta-1}\right) \cup \operatorname{dom}\left(b_{\beta-1}\right),
$$

and choose $k_{i} \in h\left(m_{j}\right)$. Having picked $\left(n_{i}, k_{i}\right)$, by letting

$$
z_{i}=y \cdot \sum_{i^{*} \leq i}\left\|\left(m_{j}, n_{i^{*}}, k_{i^{*}}\right) \in \dot{r} \backslash \dot{s}_{\beta-1}\right\|,
$$

we can use (iv), (v) and (29) with (16) to obtain $n_{i+1}>n_{i}$ satisfying (30) such that

$$
\mu\left(\left(y-z_{i}\right) \cdot g\left(n_{i+1}\right)\right)>\varepsilon \cdot \mu\left(y-z_{i}\right) .
$$

Then if we choose the best $k_{i+1} \in h\left(m_{j}\right)$ we must have

$$
\mu\left(\left(y-z_{i}\right) \cdot\left\|\left(m_{j}, n_{i+1}, k_{i+1}\right) \in \dot{r} \backslash \dot{s}_{\beta-1}\right\|\right)>\frac{\varepsilon}{h\left(m_{j}\right)} \cdot \mu\left(y-z_{i}\right) .
$$

Hence at some finite stage, i.e. $i=\left\lceil\log \left(1-\mu\left(x_{\beta-1}\right) /(2 \cdot \mu(y))\right) / \log \left(1-\varepsilon / h\left(m_{j}\right)\right)\right\rceil$, we will have that $\mu\left(z_{i}\right)>\mu\left(x_{\beta-1}\right) / 2$. Then since (30) implies that (26) holds, we are done by putting $u(j)=\left\{\left(m_{j}, n_{i^{*}}, k_{i^{*}}\right): i^{*} \leq i\right\}$. 
Now we define $a_{\beta}$ and $b_{\beta}$ by

$$
a_{\beta}=a_{\beta-1} \cup \bigcup_{j=0}^{\infty} u(j) \quad \text { and } \quad b_{\beta}=b_{\beta-1} .
$$

Condition (26) implies that (i) holds, and obviously (ii) holds. Clearly (25) and (26) imply that condition (iii) is satisfied, and since each $u(j)$ is finite, condition (iv) is also satisfied. Conditions (v) and (vi) automatically hold. And by (17), from (27) it follows that $x_{\beta-1} \cdot \| a_{\beta} \cap\left(\dot{r} \backslash \dot{s}_{\beta-1}\right)$ is infinite $\|$ has measure at least $\mu\left(x_{\beta-1}\right) / 2$ and in particular is nonzero, which implies (vii)(a).

The remaining case is

Case 2. There is an $l \in \mathbb{N}$ such that for all $m \geq l$,

$$
x_{\beta-1} \cdot\left\|\lim _{z \rightarrow \dot{\mathcal{G}}} \lim _{n \rightarrow \breve{U}} \frac{\mu(z \cdot g(m, n))}{\mu(z)}>0\right\| \text { has measure at most } \frac{\mu\left(x_{\beta-1}\right)}{2} .
$$

Let $\left\{m_{j}^{*}\right\}_{j=0}^{\infty}$ be an enumeration without repetition of all $m \in \mathbb{N} \backslash l$ such that $b_{\beta-1} \cap[\{m\} \times \mathbb{N}]=\emptyset$. By (20) and (35), for each $j$ there is an $n_{j}^{*} \in \mathbb{N}$ such that

$$
\begin{gathered}
\left(m_{j}^{*}, n_{j}^{*}\right) \notin \operatorname{dom}\left(a_{\beta-1}\right), \\
\mu\left(x_{\beta-1} \cdot\left\|\left[\left\{\left(m_{j}^{*}, n_{j}^{*}\right)\right\}\right] \cap \dot{r} \cap \dot{s}_{\beta-1} \neq \emptyset\right\|\right)>\frac{\mu\left(x_{\beta-1}\right)}{3} .
\end{gathered}
$$

Define

$$
a_{\beta}=a_{\beta-1} \quad \text { and } \quad b_{\beta}=b_{\beta-1} \cup \bigcup_{j=0}^{\infty}\left[\left\{\left(m_{2 j}^{*}, n_{2 j}^{*}\right)\right\}\right] .
$$

By (36), condition (i) holds. Conditions (ii)-(vi) are all clearly satisfied. By (17), it follows from (37) that $x_{\beta-1} \cdot \| b_{\beta} \cap \dot{r} \cap \dot{s}_{\beta-1}$ is infinite $\|$ has measure at least $\mu\left(x_{\beta-1}\right) / 3$, and in particular is nonzero, which implies (vii)(b).

Now suppose that $\beta$ is a limit. Let $\left\{\xi_{j}\right\}_{j=0}^{\infty} \subseteq \beta$ be a strictly increasing cofinal sequence. Using (ii) and (vi), recursively choose $m_{0}<m_{1}<\cdots<m_{j}<\cdots$ so that

$$
\bigcup_{i<j} b_{\xi_{i}} \cap\left[\left\{m_{j}\right\} \times \mathbb{N}\right]=\emptyset
$$

Define

$$
\begin{aligned}
a_{\beta}=\bigcup_{n=0}^{\infty} a_{\xi_{n}} \backslash & \left(\bigcup_{i<n} b_{\xi_{i}} \cup[n \times \mathbb{N}] \cup \bigcup_{i<n}\left[\operatorname{dom}\left(a_{\xi_{i}} \backslash a_{\xi_{n}}\right)\right]\right), \\
b_{\beta}=\bigcup_{n=0}^{\infty} b_{\xi_{n}} \backslash & \left(a_{\beta} \cup \bigcup_{j=0}^{\infty}\left[\left\{m_{j}\right\} \times \mathbb{N}\right]\right. \\
& \left.\cup \bigcup_{i<n} \bigcup\left\{[\{m\} \times \mathbb{N}]:[\{m\} \times \mathbb{N}] \cap\left(b_{\xi_{i}} \backslash b_{\xi_{n}}\right) \neq \emptyset\right\}\right) .
\end{aligned}
$$

Obviously condition (i) is satisfied. For each $n$, since $a_{\xi_{n}} \cap b_{\xi_{i}}$ and $a_{\xi_{i}} \backslash a_{\xi_{n}}$ are finite for all $i<n$, by (iv) only finitely many elements are being removed from $a_{\xi_{n}}$. Hence for all $\alpha<\beta, a_{\alpha} \subseteq^{*} a_{\beta}$. Also note that for each $n$, since $b_{\xi_{n}} \cap\left[\left\{m_{j}\right\} \times \mathbb{N}\right]=\emptyset$ for all $j>n$, and since $b_{\xi_{i}} \backslash b_{\xi_{n}}$ is finite for all $i<n$, by (v) only finitely many elements are being removed from $b_{\xi_{n}}$, and hence $b_{\alpha} \subseteq^{*} b_{\beta}$ for all $\alpha<\beta$. Thus 
condition (ii) holds. Since the last union in (40) guarantees that $a_{\beta}$ is a function, condition (iii) is satisfied. Noting that $a_{\beta} \cap[\{m\} \times \mathbb{N}] \subseteq \bigcup_{i \leq m} a_{\xi_{i}} \cap[\{m\} \times \mathbb{N}]$ one sees that condition (iv) is satisfied. It is clear that the last union of (41) guarantees condition (v). Finally, the set $\left\{m_{0}, m_{1}, \ldots\right\}$ witnesses that condition (vi) holds. The recursion is now complete.

\section{REFERENCES}

[AT97] Uri Abraham and Stevo Todorčević, Partition properties of $\omega_{1}$ compatible with $\mathrm{CH}$, Fund. Math. 152 (1997), no. 2, 165-181. MR 98b:03064

[Dow95] Alan Dow, More set-theory for topologists, Topology Appl. 64 (1995), no. 3, 243-300. MR 97a:54005

[Hau36] Felix Hausdorff, Summen $\aleph_{1}$ von Mengen, Fund. Math. 26 (1936), 241-255.

[Hir00a] James Hirschorn, Random trees under CH, preprint, 2000.

[Hir00b] James Hirschorn, Towers of measurable functions, Fund. Math. 164 (2000), no. 2, 165192. MR 2002i:03056

[Hir01] James Hirschorn, Summable gaps, Ann. Pure Appl. Logic 120 (2003), no. 1-3, 1-63.

[Hir03] James Hirschorn, Random gaps, preprint, October 2003.

[Jec97] Thomas Jech, Set theory, second ed., Springer-Verlag, Berlin, 1997. MR 99b:03061

[Kan94] Akihiro Kanamori, The higher infinite. Large cardinals in the set theory from their beginnings, Springer-Verlag, Berlin, 1994. MR 96k:03125

[Kun76a] Kenneth Kunen, $\left(\kappa, \lambda^{*}\right)$ gaps under MA, handwritten note, August 1976.

[Kun76b] _ Some points in $\beta N$, Math. Proc. Cambridge Philos. Soc. 80 (1976), no. 3, 385-398. MR 55:106

[Lav79] Richard Laver, Linear orders in $(\omega)^{\omega}$ under eventual dominance, Logic Colloquium '78 (Mons, 1978), North-Holland, Amsterdam, 1979, pp. 299-302. MR 81e:03051

[Sch93] Marion Scheepers, Gaps in $\omega^{\omega}$, Set theory of the reals (Ramat Gan, 1991), Bar-Ilan Univ., Ramat Gan, 1993, pp. 439-561. MR 95a:03061

[Sol71] Robert M. Solovay, Real-valued measurable cardinals, Axiomatic set theory (Proc. Sympos. Pure Math., Vol. XIII, Part I, Univ. California, Los Angeles, Calif., 1967), Amer. Math. Soc., Providence, R.I., 1971, pp. 397-428. MR 45:55

[TF95] Stevo Todorčević and Ilijas Farah, Some applications of the method of forcing, Yenisei, Moscow, 1995. MR 99f:03001

[Tod89] Stevo Todorčević, Partition problems in topology, American Mathematical Society, Providence, RI, 1989. MR 90d:04001

[Tod00] - A dichotomy for P-ideals of countable sets, Fund. Math. 166 (2000), no. 3, 251-267. MR 2001k:03111

[Woo84] W. Hugh Woodin, Discontinuous homomorphisms of $C(\Omega)$ and set theory, Ph.D. thesis, University of California, Berkeley, 1984.

Department of Mathematics, University of Helsinki, Helsinki, Finland

Current address: Centre de Recerca Matemàtica, Apartat 50, E-08193 Bellaterra, Spain

E-mail address: jhirschorn@crm.es

E-mail address: James.Hirschorn@logic.univie.ac.at

URL: http://www.logic.univie.ac.at/〜hirschor/ 